\title{
2D numerical simulation of ocean waves
}

\author{
Qingjie. Dư*, Y.C. Dennis. Leung \\ Department of Mechanical Engineering, The University of Hong Kong, Hong Kong, China \\ *Corresponding author. Tel: +852 51743593, E-mail: qingjie.du@gmail.com
}

\begin{abstract}
As fossil energy is depleting and global warming effect is worsening rapidly, developing renewable energies become the top priority on most developed and some developing countries. Among different kinds of renewable energies, wave energy attracts more and more attention in recent years due to its high energy density and enormous global amount. However, some technical difficulties still need to be overcome for extracting wave power. In designing a wave energy converter, it is important to develop an efficient method to determine the wave load and predict its response. In this paper, a n umerical investigation of ocean waves is presented. Commercial software code FLUENT is used as a computational platform in this study. Based on the NavierStokes equations for viscous, incompressible fluid and Volume of fluid (VOF) method, a two dimensional numerical wave tank is established. Dynamic meshing method is used to simulate the wave maker, and GeoReconstruct scheme is used to capture the free surface. A wave-absorbing method employing porous media model is proposed, which can absorb the wave energy efficiently. Moving boundary, wall boundary and pressure-inlet boundary are used to construct the computational domain. Linear regular waves are simulated accurately using the proposed numerical model. The numerical results matched with the theoretical calculation.
\end{abstract}

Keywords: Numerical wave flume, FLUENT, VOF method, Dynamic meshing

\section{Nomenclature}

\begin{tabular}{|c|c|c|c|}
\hline$u$ & velocity component ( $x$-direction) ..........m $\cdot^{-1}$ & $S$ & paddle stroke.. \\
\hline$v$ & velocity component (y-direction) ..........m $\cdot^{-1}$ & $T$ & wave period.... \\
\hline$\rho$ & 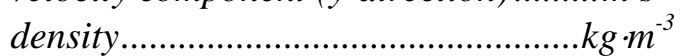 & $k$ & wave number \\
\hline$\mu$ & dynamic viscosity ............................ $\mathrm{kg} \cdot \mathrm{m}^{-\mathrm{s}^{-1}}$ & $h$ & 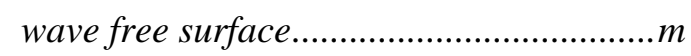 \\
\hline ? & static pressure........... & $\omega$ & angular frequency ........................................... $\mathrm{s}^{-1}$ \\
\hline$a$ & body force $(x$-direction)... & $f$ & 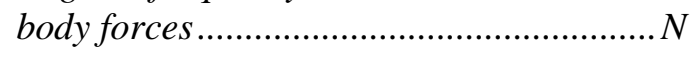 \\
\hline
\end{tabular}

\section{Introduction}

The World Energy Council (1999) reported that the total globally extractable wave energy is about 2 Terawatts [1], which is the same order of magnitude as the world's total electricity consumption. How to harness this huge energy has attracted more and more scientists' attention. In the design of wave energy converter, predicting wave loads and the structure responses have become increasingly important. In the past, the study of wave-structure interaction is mainly based on physical model experiment, which is both time consuming and money costly. Nowadays, following the rapid development in computational method and computer hardware, numerical simulation of the wave-structure interaction has attracted more and more attention.

The computation of unsteady free-surface flow is a key point in two-phase flow. Hirt and Nichols [2] developed the Volume of fluid (VOF) method to solve the two-phase problem, which uses a geometrical reconstruction scheme to capture the free surface. Wang et al [3] employed a numerical method to simulate the wave group development in long tanks. Zou [4] and Liu [5] used a moving boundary to simulate the piston-type wave maker, and successfully generated regular waves. Wei et al [6] and Chawla [7] implemented a source function method to generate ocean waves, based on Boussinesq model. Based on the 2D form of Navier-Stokes 
equations, Dong and Huang [8] established a 2 D numerical wave tank to simulate smallamplitude waves and solitary waves. Lu et al [9] numerically simulated wave overtopping against seawalls in regular wave case.

During the last two decades, numerous scientists have developed their numerical methods to simulate ocean waves that are nonlinear and unsteady free-surface flows. In this paper, the research is focused on the simulation of a two-dimensional numerical wave flume. FLUENT is used as the main computational platform. Some User-Define-Function (UDF) has been implemented to simulate the wave maker and wave absorbing bench. Dynamic meshing technique is used to simulate a piston-type wave maker, which can generate both regular and irregular waves. VOF model is used to capture the free surface between water and air. Porous media model acts as the wave absorbing bench to absorb the wave energy. Both linear and nonlinear waves are simulated and compared with theoretical result.

\section{Governing equation}

In fluid dynamic research, there are several important assumptions. F irst, the fluid being studied is assumed to be a continuum; second, all field involved are differentiable, such as velocity field, pressure field. Moreover, for the water fluid dynamic field, some other sound assumptions are also established. Water is assumed to be Newtonian fluids, and it is incompressible and its density will not change with time. Based on the above assumptions, the Navier-Stokes equation and continuity equation are used to describe the fluid motion, which are also the governing equations in this study.

\subsection{Navier-Stokes equation}

Equation 1 shows the Navier-Ssokes equation in vector form:

$\rho\left(\frac{\partial v}{\partial t}+v \cdot \nabla\right)=-\nabla p+\mu \nabla^{2} v+f$

Rewriting the vector equation explicitly in 2D Cartesian coordinates:

$$
\begin{aligned}
& \rho\left(\frac{\partial u}{\partial t}+u \frac{\partial u}{\partial x}+v \frac{\partial u}{\partial y}\right)=-\frac{\partial p}{\partial x}+\mu\left(\frac{\partial^{2} u}{\partial x^{2}}+\frac{\partial^{2} u}{\partial y^{2}}\right)+\rho g_{x} \\
& \rho\left(\frac{\partial v}{\partial t}+u \frac{\partial v}{\partial x}+v \frac{\partial v}{\partial y}\right)=-\frac{\partial p}{\partial y}+\mu\left(\frac{\partial^{2} v}{\partial x^{2}}+\frac{\partial^{2} v}{\partial y^{2}}\right)+\rho g_{y}
\end{aligned}
$$

\subsection{Continuity Equation}

Equation 3 shows the continuity equation in 2D Cartesian coordinates

$$
\frac{\partial u}{\partial x}+\frac{\partial v}{\partial y}=0
$$

\section{Numerical method}

\subsection{Boundary condition}

Given proper boundary condition and initial condition, the above equations can be solved computationally. For a $2 \mathrm{D}$ case, given the velocity potential $\Phi$, the boundary conditions are as follows:

(a) dynamic free-surface condition: 
$\frac{\partial \phi}{\partial t}=-g \eta-\frac{1}{2}|\nabla \phi|^{2}-\frac{p_{0}}{\rho}$

where $\mathrm{P}_{0}$ is the pressure on the free surface.

(b) kinematic free-surface condition:

$\frac{\partial \eta}{\partial t}=-\nabla \phi \cdot \nabla \eta+\frac{\partial \phi}{\partial y}$

(c) No normal-flux condition:

$\frac{\partial \phi}{\partial n}=0$

applied on the rigid bottom, and at the vertical end-wall of the numerical flume.

In FLUENT, boundary condition (a) and (b) is satisfied by using the VOF scheme, and boundary condition (c) is satisfied by using wall condition.

\subsection{Free surface--VOF model}

The main goal of the present research is to study the characteristics of ocean waves under different scenarios. In this research, the key problem is how to accurately describe the free surface between the two different phases. In this paper, since all the calculation is based on FLUENT, VOF model is used to simulate the free surface between water and air. The method is based on the idea of so called fraction function $\alpha_{q}$. It is defined as the integral of fluid's characteristic function in the control volume (namely volume of a computational grid cell). Basically, when the cell is empty, $\alpha_{q}=0$; if the cell is full, $\alpha_{q}=1$; if $0<\alpha_{q}<1$, then the volume is the interface between the two phases. For this study, it is a two-phase problem, so $\mathrm{q}=1,2$, representing air and water respectively. $\mathrm{q}$ is tracked by solving the continuity equation below for $\mathrm{q}^{\text {th }}$ fluid.

$\frac{\partial x_{q}}{\partial t}+\frac{\partial\left(u x_{q}\right)}{\partial t}+\frac{\partial\left(v \alpha_{q}\right)}{\partial t}=0$

$\sum_{q=1}^{2} \alpha_{q}=1$

\subsection{Wave maker--dynamic mesh}

A piston-type wave maker is simulated using the dynamic mesh technology in this study. Moving boundary is used to model the oscillating paddle in the physical wave maker. A userdefined function (UDF) is used to describe the motion of the oscillating paddle. In order to make the simulation more smoothly, the velocity of the paddle is described as follows:

$$
\begin{array}{ll}
\mathrm{U}=\frac{\mathrm{t}}{2 \mathrm{~T}} \frac{\mathrm{s}}{2} \omega \cos (\omega \mathrm{t}) & \mathrm{t} \leqq 2 \mathrm{~T} \\
\mathrm{U}=\frac{\mathrm{s}}{2} \omega \cos (\omega \mathrm{t}) & \mathrm{t}>2 \mathrm{~T}
\end{array}
$$


where $S$ is the stroke of the paddle, $t$ is the run time, $T$ is the wave period, $\omega(\omega=2 \pi / T)$ is the angular frequency.

\subsection{Wave absorbing bench--Porous media}

The mathematic model of the porous medium is defined as:

$S_{j}=-\left(\frac{\mu}{\alpha} v_{j}+C_{2} \frac{1}{2} \rho|v| v_{j}\right)$

where $\mathrm{i}$ represents $\mathrm{x}, \mathrm{y}, \mathrm{z} ; \frac{1}{\alpha} \& \mathrm{C}_{2}$ are the coefficients of viscous resistance $\&$ inertial resistance respectively.

In this $2 \mathrm{D}$ model, only $\frac{1}{\alpha}$ is considered. How to decide the value of $\frac{1}{\alpha}$ is very important. If $\frac{1}{\alpha}$ is too small, the wave energy cannot be absorbed completely and when waves reach the right boundary, it will reflect. If $\frac{1}{\alpha}$ is too large, the fluid property will change dramatically in the interface between the water and porous medium, so the wave will reflect too. In this simulation, the coefficient is defined as a function of the position, which is described by a $\mathrm{UDF}$, in order to make the resistance of the porous medium change smoothly.

\subsection{Computational model}

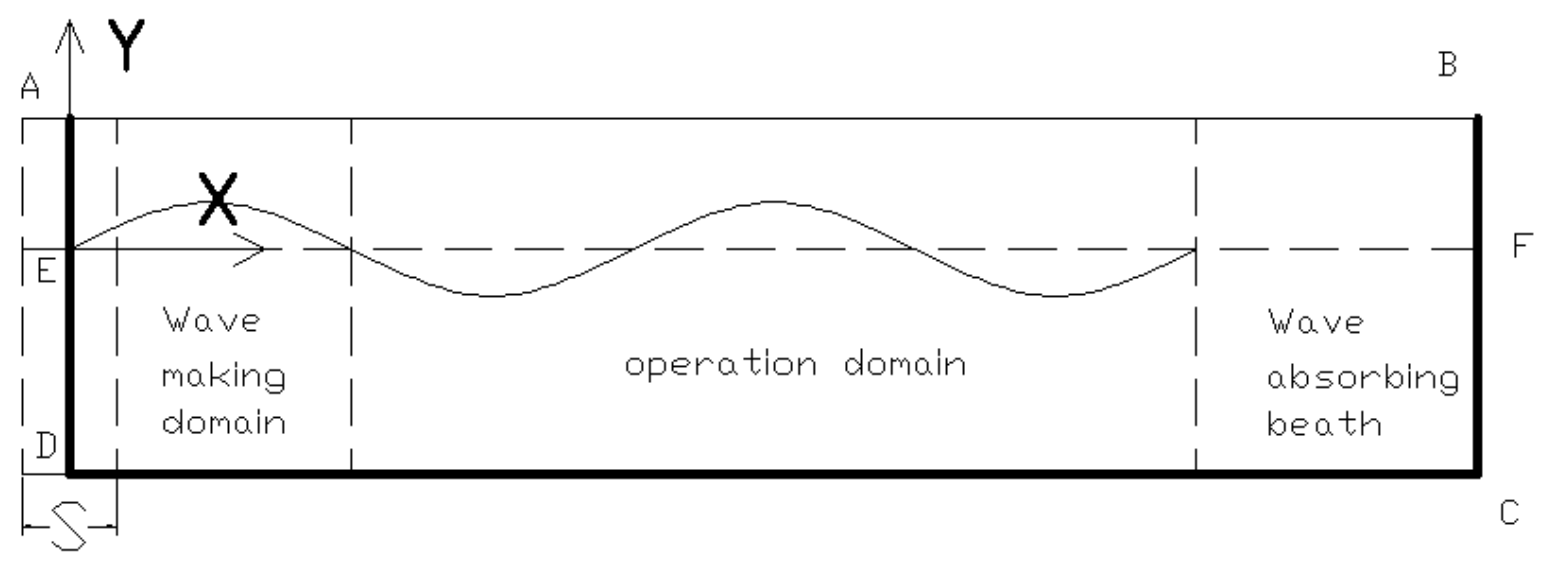

Fig.1 computational model scheme

Fig. 1 shows a computation scheme of this study. Boundary AD represents the oscillating paddle; Boundary DC represents the wave flume bottom; Boundary BC represents the end of the wave flume; Boundary AB represents the top of the flume. The Volume of Fluid model is used to describe the free surface between the air and water.

\section{Results and discussion}

In this study, the numerical model is based on the dimension of a physical wave flume in the laboratory, with a dimension of $9 \mathrm{~m}$ long and $0.45 \mathrm{~m}$ high. In this simulation, the initial water depth is $0.3 \mathrm{~m}$. A linear regular wave with a wave height at $0.05 \mathrm{~m}$ and wave period at $1.8 \mathrm{~s}$ will be simulated by the numerical model. The theoretical analysis will be performed too. The wave form in the whole tank is monitored at certain times. Also the surface elevation history at point $\mathrm{x}=4.5 \mathrm{~m}$, which is the center point of the physical wave tank, is monitored too. These data will be compared with the theoretical result. 
Under the assumption of both small amplitude paddle motion and small wave height, linear wavemaker theory has been developed by Dean and Dalrymple [10]. Assuming the original place of the paddle is at $\mathrm{x}=0$, and the wavemaker stroke is $\mathrm{S}$, the angular frequency of the paddle is $\omega$, the wave elevation on the free surface $\eta$ in the wave tank with water height $d$ is shown as follows:

$$
\begin{aligned}
& \eta=\frac{s}{2}\left[\frac{4 \sinh h^{2}(k d)}{2 k d+\sinh (2 k d)} \cos (k x-\omega t)\right] \\
& \omega^{2}=g k \tanh (k d)
\end{aligned}
$$

Fig. 2 shows the water elevation history at $\mathrm{x}=4.5 \mathrm{~m}$ from the time of $40 \mathrm{~s}$ to $50 \mathrm{~s}$. $40 \mathrm{~s}$, which is about 20 wave periods, is thought to be long enough for the wave to be fully developed. The black line with stars represents for the numerical result, and the green dotted line represents for the theoretical result.
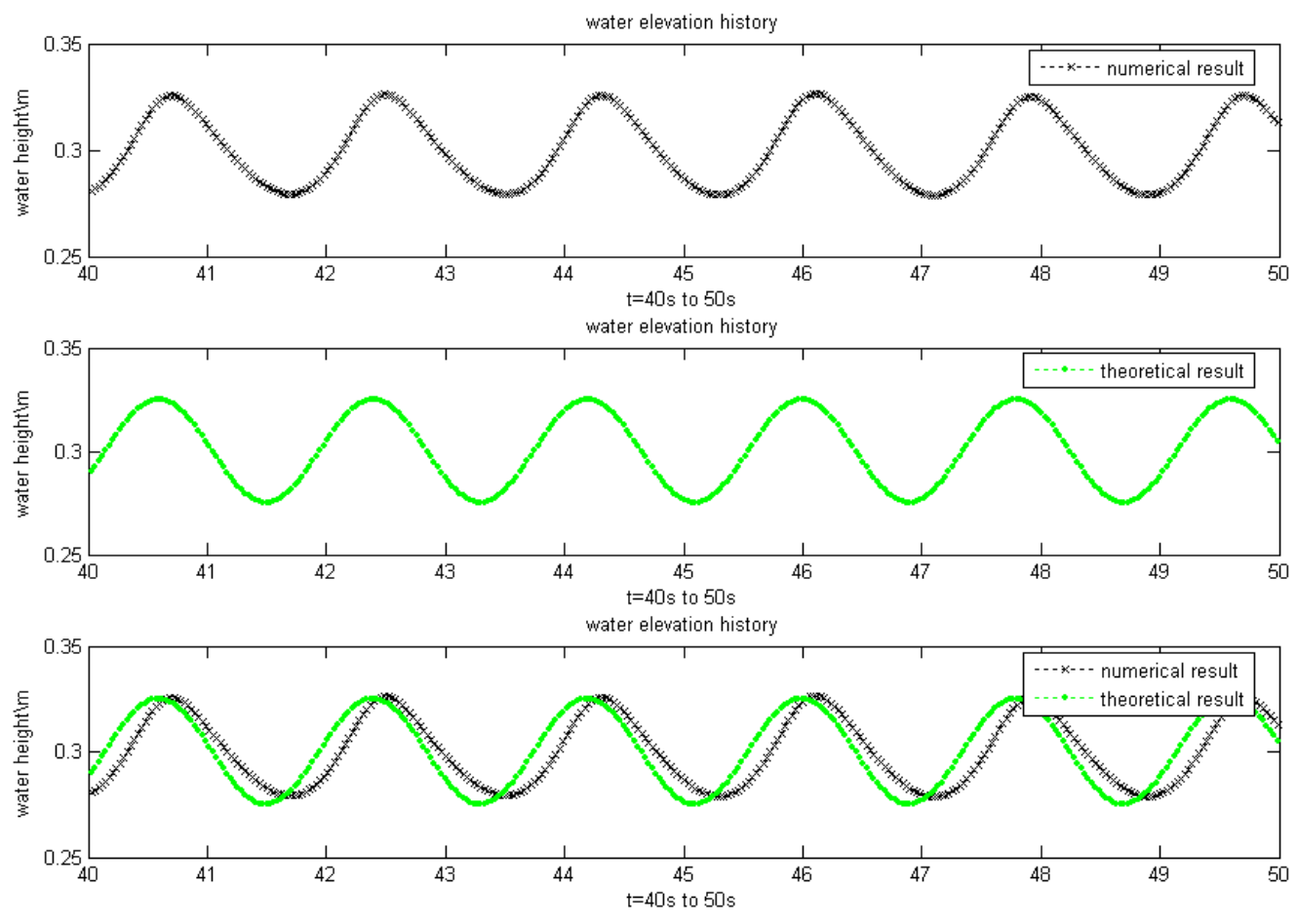

Fig. 2 surface elevation history at point $x=4.5$

Though there is a slight shift in the phase, the numerical result matches the theoretical result well. The phase shift is due to the first 2 periods. In the simulation, during first 2 periods, all the parameters were dividing by $2 \mathrm{~T}$ for smoothing issue.

Fig. 3 shows the water free surface at $\mathrm{t}=40 \mathrm{~s}, 45 \mathrm{~s}$ and $50 \mathrm{~s}$. The water surface is sinusoidal, which matches the theoretical analysis. 


\section{$\mathrm{t}=40 \mathrm{~s}$}
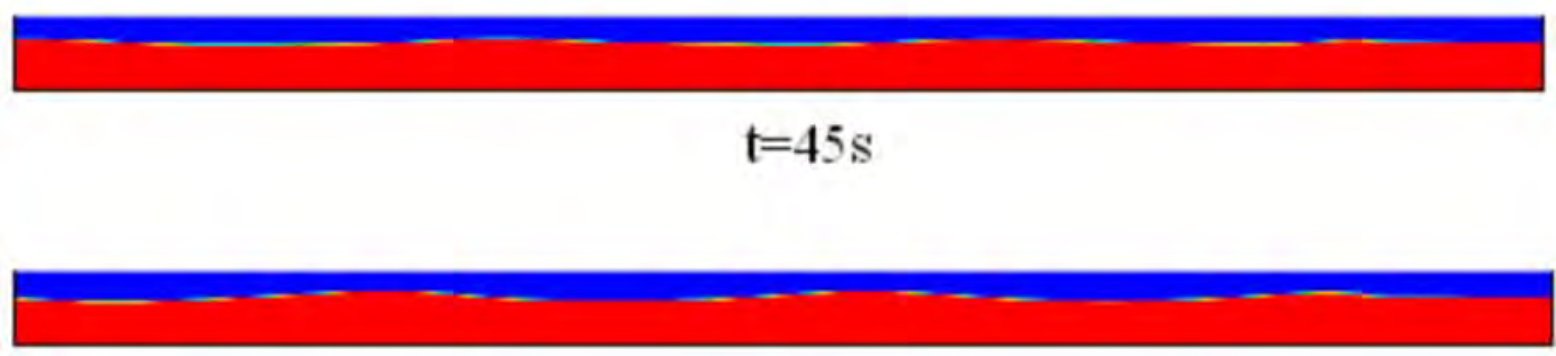

\section{$\mathrm{t}=50 \mathrm{~s}$}

Fig. 3 wave surface at $t=40$ s, $45 \mathrm{~s}$, 50 s

Noted that for the wave absorbing model, the waves are absorbed completely by the porous media in the right part. Otherwise, the waves will reflect since the right boundary condition is wall.

\section{Conclusion}

In this study, the wave simulation model is developed based on a commercial software FLUENT for modeling fluid flow. Linear regular waves and wave absorbing bench are simulated well with a self-developed numerical model. The simulation of a wave with a wave height of $0.05 \mathrm{~m}$ and period of $1.8 \mathrm{~s}$ is conducted successfully. Comparison between the numerical and theoretical results shows that the numerical method works well.

Compared with the physical model experiments, this numerical model is more adaptable. The wave tank dimension can be changed according to the specific situation. The use of this numerical model is costless and is very convenient.

Based on this model, the wave-structure interaction can be studied. What's more important is that it can serve as a platform to predict the hydrodynamic response of wave energy convertors (WECs) in waves, and the result can be used to optimize the WECs.

\section{Acknowledgement}

The authors wish to acknowledge the IEEE of the University of Hong Kong for supporting the project.

\section{References}

[1] Thorpe, T.W., A brief review of wave energy. ETSU Report Number R-120 for the DTI, 1999.

[2] Hirt, C.W. and B.D. Nichols, Volume of fluid (VOF) method for the dynamics of free boundaries. Journal of Computational Physics, 1981. 39(1): pp. 201-225.

[3] Wang, P., Y. Yao, and M.P. Tulin, Wave group evolution, wave deformation, and breaking: simulations using LONGTANK, a numerical wave tank. International Journal of Offshore and Polar Engineering, 1994. 4(3): pp. 200-205. 
[4] Zou, Z., Numerical simulation of nonlinear wave generated in wave flume by VOF technique. Journal of Hydrodynamics, 1996. 11(1): pp. 93-103.

[5] LIU, J.H., Making waves in 2-d numerical flume and feature analysis of the numerical waves. Journal of Sichuan University, 2004. 36(6): pp. 28-31.

[6] Wei, G., J.T. Kirby, and A. Sinha, Generation of waves in Boussinesq models using a source function method. Coastal Engineering, 1999. 36(4): pp. 271-299.

[7] Chawla, A. and J.T. Kirby, A source function method for generation of waves on currents in Boussinesq models. Applied Ocean Research, 2000. 22(2): pp. 75-83.

[8] Dong, C.M. and C.J. Huang, Generation and propagation of water waves in a t wodimensional numerical viscous wave flume. Journal of Waterway, Port, Coastal and Ocean Engineering, 2004. 130(3): pp. 143-153.

[9] Lu, Y.j., et al., Numerical simulation of two-dimensional overtopping against seawalls armored with artificial units in regular waves. Journal of Hydrodynamics, 2007. 19(3): pp. 322-329.

[10] Dean, R.G. and R.A. Dalrymple, Water wave mechanics for engineers and scientists. 1984. 